\title{
Interference Issues in Future Mobile Networks
}

\author{
Shafi Ullah Kamran \\ University of Engineering and Technology Peshawar \\ Department of Computer Systems Engineering \\ Main Campus, 25120 Peshawar
}

\begin{abstract}
Interference is a major impediment in communications via cellular radio networks. Future networks such as Long Term Evolution-Advanced (LTE-A) and 5G networks are no exception and interference issues are liable to degrade network performance drastically. This paper investigates methods for interference mitigation in existing mobile communication standards and proposes a novel technique for reduction of interference. A dynamic transmit power manipulation method is proposed in this work which monitors interference levels in the cellular traffic through Channel Quality Indicator (CQI) reports and maneuvers the device transmit powers according to threshold values based on Signal-to-Interference-and-Noise-Ratio (SINR). A simulation model for the proposed framework is developed to evaluate system performance and results collection.
\end{abstract}

\section{Keywords}

Interference, simulation, SINR, small cell

\section{INTRODUCTION}

Future mobile networks are being designed due to the fact that current networks have reached limits of capacity. The usage of mobile devices is increasing with each passing day and this trend is going to continue in the coming years. Service providers are generally concerned about good signal strengths at the receiver end. However, increasing transmit power can also result in increased interference levels in neighboring cells. Research community has long been looking for ways to overcome issues caused by interference. One such method is to divide coverage area into smaller cells e.g. femtocell, picocell etc. Small cells are very handy in indoor propagation environments. The small cell base stations are usually small in size, low in power consumption and cost effective. However, in some situations, such base stations can also cause interference to the signals of normal base stations. Handling interference in small cells is the primary focus of this paper. Small cell deployment without proper planning is not recommended and interference issues can be only solved if adequate planning is in place.

\section{LITERATURE REVIEW}

Currently available scientific literature on small cells architecture reveals that the research area is still quite hot and significant work remains to be done. The authors in [1] suggest an approach that has been applied for exploitation of resources in densely populated traffic environment. The methods suggested in [1] mainly deal with the status of user for small cell categorization. In [2], the authors have proposed two schemes of cell selection for managing handover issues in small cells. In [3], the authors have addressed small cell functions and designed an advanced methods related to access mode. In [4], a model has been proposed for indoor as well as outdoor propagation environment in buildings. Random indoor deployment of transmitters according to building structure has been suggested. Another method has been suggested in [5] for efficient spectrum allocation to macrocell users in vicinity of small cell. Results show promising performance.

In [6], the authors have suggested a spectrum sharing technique which is shown to be very useful for throughput performance of small and macro cell users. In [7], authors propose a power economic mechanism and analyse the scheme to show that heavily deploying low power small cells can influence power spending. In [7], it is also suggested that cloud radio mechanism of access network can respond in case of changes in traffic situation so that minimum power is consumed. In [8], a new power friendly method is designed and analysed for sparsely built small cells with power control for making heterogeneous networks less power consuming. In [9], a method has been devised for small cells where a compromise point has to be reached to encourage owners of small cells to share resources of access point to enhance efficiency. In [10], authors have designed a policy for spectrum allocation to enforce optimal utilization of small cell by implementing hybrid policy for access to network and identify three classes i.e. non-subscriber, subscriber and user of other network. Rules of operation are based on some policy for spectrum allocation. In [11], authors have studied the influence of discharging outdoor users of macro cell by handing them over to small cells inside coverage area of small cell. The authors of [12] have proposed a method where a cluster of small cells is given the control of local users and the base station is given the authority of monitoring the users' ability of detecting interference limitations of neighbouring small cell because spectrum may be reused spatially for avoiding interference among small cells.

\section{PROBLEM STATEMENT}

In mobile networks, downlink interference is generally caused by small cells after deployment alongside macrocell where both are using the same band of licensed spectrum. Small cell base station is required to send signals with transmit power levels of magnitude such that acceptable Signal-toInterference-and-Noise-Ratio (SINR) values are achieved. However, this would also result in degradation of macro downlink signal quality due to interference from small cell.

Macro users would be bound to suffer from such signals when receiving signals coming in downlink direction. Future networks like Long Term Evolution-Advanced (LTE-A) and $5 \mathrm{G}$ can pose extremely severe challenges in managing interference caused by small cells.

In $5 \mathrm{G}$, such issues could turn out to be much more severe due to the nature of (expected) air interface schemes that are anticipated to be standardized by the 3rd Generation Partnership Project (3GPP). 5G could offer access methods with flexible Physical Resource Block (PRB) sizes and sparsely coded multiple access schemes. These new air 
interface schemes are prone to interference and some level of immunity is need of the hour; otherwise, small concept may not flourish as expected in future.

Table 1. Settings for Simulation

\begin{tabular}{|c|c|}
\hline Setting & Amounts \\
\hline Macro transmission power & $46 \mathrm{dBm}$ \\
\hline Small transmission power (initial) & $0 \mathrm{dBm}$ \\
\hline Small transmission power (max.) & $10 \mathrm{dBm}$ \\
\hline Macrocell layout & 1 cell, omni \\
\hline Downlink bandwidth & $25 \mathrm{PRBs}$ \\
\hline Small and macrocell distance & $250 \mathrm{~m}$ \\
\hline Max. small cell radius & $50 \mathrm{~m}$ \\
\hline Minimum coupling loss & $70 \mathrm{~dB}$ \\
\hline Penetration losses due to walls & $17 \mathrm{dBm}$ \\
\hline Macrocell radius & $1500 \mathrm{~m}$ \\
\hline Path loss model $(R$, distance in km) & $128.1+37.6 \log _{10}(R)$ \\
\hline Slow fading & Lognormal, STD $8 \mathrm{~dB}$ \\
\hline Mobility model & Random way point \\
\hline
\end{tabular}

\section{PROPOSED METHOD}

A scheme for mitigation of interference caused by small cells in mobile networks is proposed here in this work. The scheme is based on a solution designed with emphasis on idiosyncrasies of both LTE-A and 5G networks. The scheme operates on the principle of power management for interference control of signals from small cell base station to macro users.

The scheme utilizes the mechanism of feedback signals in the form of Sounding Reference Signals (SRS) to estimate or gauge the levels of interference at the macrocell environment for macro users.
The proposed scheme also keeps an eye on the utilization of radio resources. Resource allocation situation is then fed back to the small cell, which contains information about SINR levels as well as PRB allocation status in the macrocell.

Performance goals set as benchmark for this scheme are:

- Improved SINR values

- Improved cell capacity

The transmission power of small cell changes dynamically depending upon the feedback obtained regarding interference level at the macro cells. This role of SRS is fully function and utilized to acquire the required input data for the scheme. Based on input, transmission power is modified accordingly.

The proposed mechanism is implemented using simulation environment where a server node is placed to model the features of core network. A base station is placed in the radio access part of the network and user nodes are connected to this base station for cellular coverage. A small cell is also placed in the radio access part with users also connected to it. Five users near macrocell are served by macro base station while five users near small cell are served by small cell base station.

A router is also placed for routing packets from both macro base station and small base station which are, thus, connected to each other and the core network, while exchange of data is also facilitated. Router settings are tuned as per Internet Protocol (IP) model and routing protocols. In accordance with 3GPP standards, the access network part is also developed and designed in this work. A central register containing information of base stations and connected users is also maintained. It is the pivotal and principal database with records of each user and base station. It collects and apprises information for all mobile devices with corresponding serving base station information as well as mobility information.

This simulation model thrives upon a Proportional Fair (PF) resource allocation strategy for distribution of frequency and time based resources inside macro and small cells respectively. Table 1 shows the simulation parameters applied in this work.

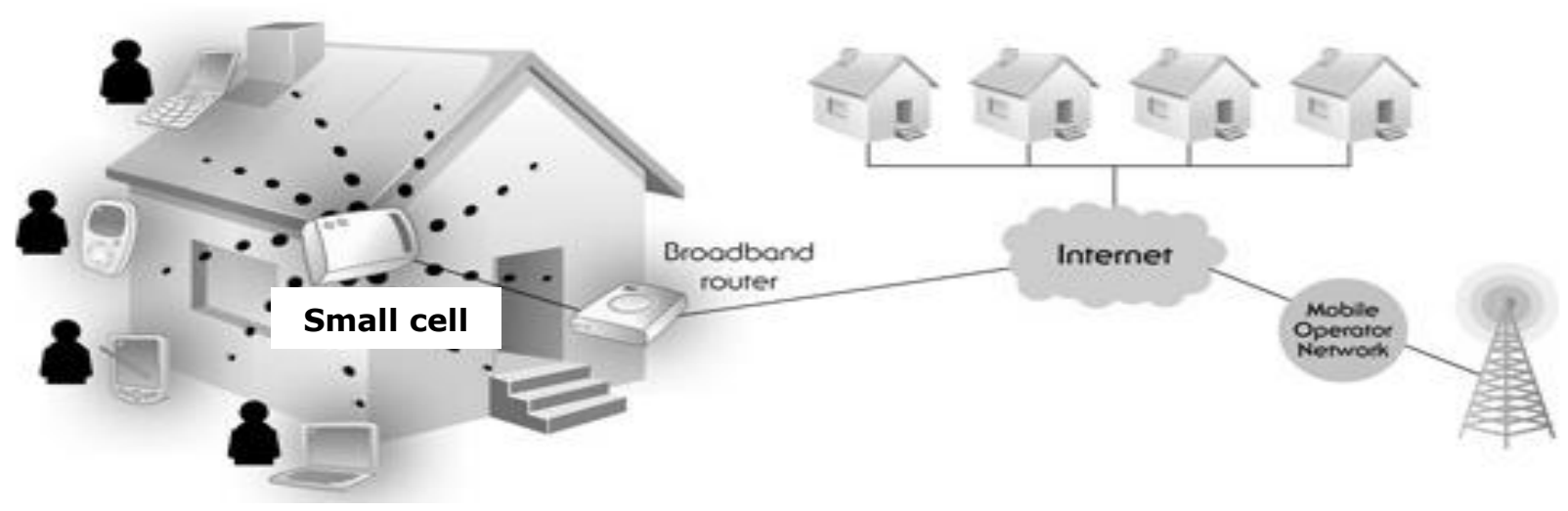

Fig 1: Typical indoor small cell [13]

\section{RESULTS}

Performance evaluation of macro and small cell users is achieved by comparing SINR performance in simulation scenarios. The performance is depicted by capturing SINR values at various transmit power levels of small base station which is dynamically adapted as per feedback from macro cell. As observed, increase in transmit power reduces SINR level for macro users. But in contrast, increase in transmit power improves transmit power for small cell users. When transmit power is maximum, signal quality improves significantly for small cell users. Hence, a tradeoff is successfully established. 


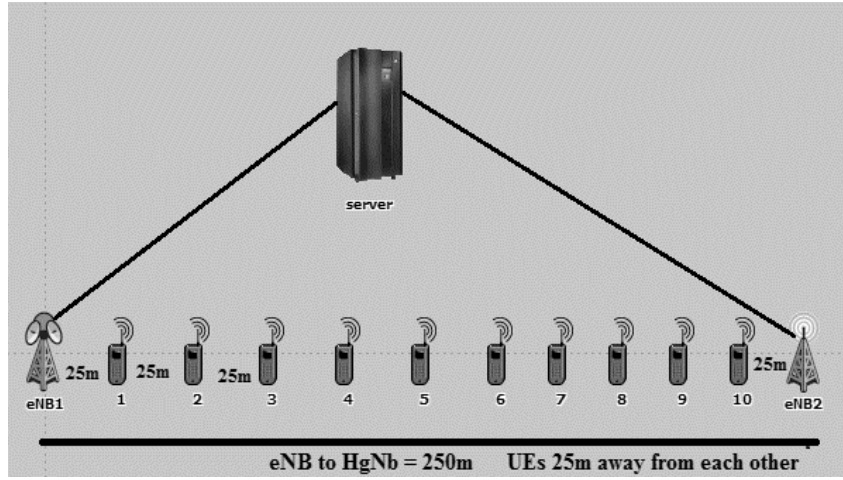

Fig 2: Simulation environment

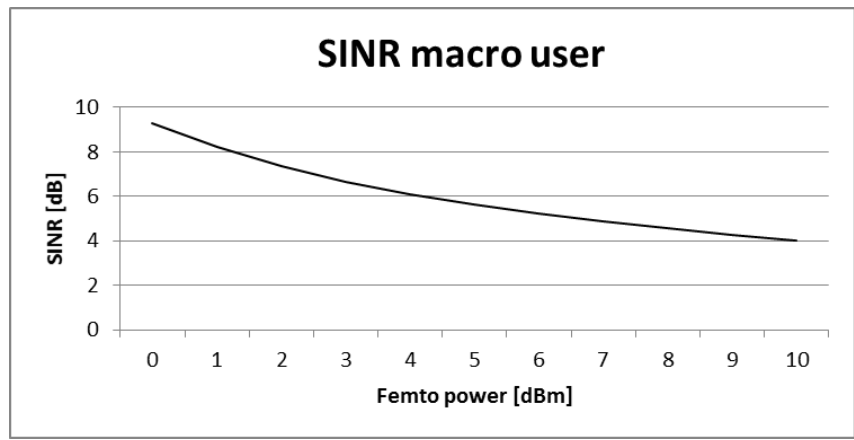

Fig 3: Macro cell user results

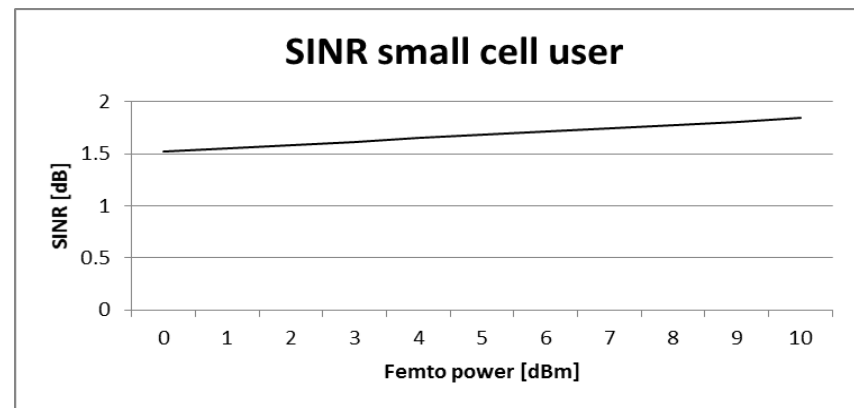

Fig 4: Small cell user results

\section{CONCLUSION}

A simulation environment is utilized for design, implementation and analysis of proposed power manipulation based scheme where the transmit power of small cell base station is dynamically adapted according to feedback from macro cell environment. Transmit power of small cell is modified as per situation for interference mitigation. Results reveal that a tradeoff between macro and small cell user's SINR is corroborated

\section{ACKNOWLEDGMENTS}

I am extremely thankful to University of Engineering and Technology, Peshawar for supporting this work by providing simulation tools.

\section{REFERENCES}

[1] I. Shgluof, M. Ismail and R. Nordin, "Semi-Clustering of Victim-Cells Approach for Interference Management in Ultra-Dense Femtocell Networks," IEEE Access, vol. 5, pp. 9032 - 9043, 19 Apr. 2017.
[2] N. Hoang and N. Nguyen, "Cell Selection Schemes for Femtocell-to-Femtocell," in Keattisak Sripimanwat National Electronics and Computer Technology Center, Hanoi, Vietnam, 22-25 Oct. 2014

[3] N. K. Bao, S. Joung and M. Park, "A new access mode for femtocells in 5G networks," in International Conference on Information and Communication Technology Convergence, Jeju, South Korea, 28-30 Oct. 2015.

[4] S. Hamid, K. Mubarak and A. Shami, "Inside-Out Propagation: Developing a Unified Model for the Interference in 5G Networks," pp. 47 - 54, 23 April 2015.

[5] C. Bouras, G. Diles and V. Kokkinos, "Femtocells coordination in future hybrid access deployments," in 2014 11th International Symposium on Wireless Communications Systems (ISWCS), Barcelona, Spain, 26-29 Aug. 2014

[6] P. Mach and Z. Becvar, "Distributed Hybrid Spectrum Sharing for OFDMA-based Cognitive Femtocells in 5G Networks," in Proceedings of European Wireless 2015; 21th European Wireless Conference, Budapest, Hungary, 20-22 May 2015.

[7] A. Dulaimi, A. Anpalagan, M. Bennis and A. Vasilakos, "5G Green Communications: C-RAN Provisioning of CoMP and Femtocells for Power Management," in IEEE International Conference on Ubiquitous Wireless Broadband (ICUWB), Montreal, QC, Canada, 2015.

[8] M. Haddad, and M. Bayoumi, "Green novel power control framework for dense femtocell grids," in International Conference on Computer Vision and Image Analysis Applications, Sousse, Tunisia, 18-20 Jan. 2015.

[9] N. Bao,, S. Joung, and M. Park, "A new access mode for femtocells in 5G networks," in International Conference on Information and Communication Technology Convergence (ICTC), Jeju, South Korea, 28-30 Oct. 2015.

[10] C. Bouras and G. Diles, "Resource management in 5G femtocell networks," in International Conference on Broadband and Wireless Computing, Communication and Applications (BWCCA), Krakow, Poland, 4-6 Nov. 2015.

[11] M. Khan, F. Bhatti, A. Habib, S. Jangsher and M. Khan, "Analysis of macro user offloading to femto cells for 5G cellular networks," in International Symposium on Wireless Systems and Networks (ISWSN), Lahore, Pakistan, 2017.

[12] H. Wang, E. Dutkiewicz, G. Fang and M. Mueck, "A fair spectrum sharing framework based on topological sort and max weight for femtocells," in Australian Communications Theory Workshop (AusCTW), Melbourne, VIC, Australia, 20-22 Jan. 2016.

[13] L. Zhang, L. Yang and T. Yang, "Cognitive Interference Management for LTE-A Femtocells with Distributed Carrier Selection," in Vehicular Technology Conference Fall (VTC 2010-Fall), 2010 IEEE 72nd, 2010. 